\title{
Rethinking endometriosis and pelvic pain
}

\author{
Kristin Patzkowsky
}

Department of Gynecology and Obstetrics, Johns Hopkins School of Medicine, Baltimore, Maryland, USA.

$E$ ndometriosis is defined as the presence of endometrial glands and stroma outside of the uterine cavity that can lead to pelvic pain and infertility (1). Clinical histories consistent with endometriosis can be found dating back to the 17th and 18th centuries (2). In 1899, Russel first described and illustrated the presence of endometrial tissue within an ovary (3). In 1921, Sampson published his first case series of 23 patients with ovarian endometriomas and posited the theory of retrograde menstruation (4). Endometriosis affects up to $10 \%-15 \%$ of women of reproductive age (1). Despite this prevalence and ongoing research efforts, much about endometriosis remains an enigma, including its variability in symptoms and progression of disease, underlying pain mechanisms, effect on fertility, and response to treatment.

After more than one hundred years of experience with endometriosis, why do we understand so little? To move the field forward, perhaps it's time to take a step back, pause, and reconsider critical issues related to endometriosis.

\section{What's in a name?}

In medical school, we are taught the "classic" phenotype of endometriosis - early onset of severe menstrual period pain (dysmenorrhea) that often progresses to include noncyclic pain - as well as the 3 “dys's," i.e., dyschezia (pain when defecating that may be accompanied by changes in frequency of bowel movement), dyspareunia (persistent or recurrent vaginal pain that occurs during or after intercourse), and dysuria (pain associated with urination) (1). These symptoms characterize many women with endometriosis, but teaching this narrow clinical presentation alone is woefully inadequate and a disservice to our patients. I, too, was taught this dictum, but after years of treating patients with endometriosis, I have learned that endometriosis presents in any way it wants - with any degree, character, type, or location of pain. Patients with endometriosis may present with no pain at all, at any point in the reproductive age spectrum, with or without preceding symptoms, and may have only nongynecologic symptoms.

Conversely, not all pelvic pain indicates endometriosis or is even gynecologic. To assume that all women with this classic phenotype have endometriosis and then to aggressively and narrowly treat them without considering other etiologies is another grave disservice. We need to approach these patients with the knowledge that there are multifactorial etiologies of pelvic pain including, but not limited to, functional bowel disorders, pelvic floor dysfunction, interstitial cystitis, and neuropathic pain. Further, it is important to recognize and address the complex ways that mental illnesses, such as depression and anxiety, can affect the response to treatment of chronic pain (5). For providers who encounter women with pelvic pain, it is critical for timely diagnosis and management to consider gynecologic and nongynecologic causes as well as understand that there are visceral and somatic origins of pain (6).

\section{Research in endometriosis}

We have some understanding of the different mechanisms of pain causation in endometriosis, but our understanding of "who" has pain and "how" pain occurs is overall very poor (6). When does endometriosis stop being a normal consequence of menstruation and become a disease (6)? There is excellent research being done on endometriosis, but the definition of these key characteristics is often muddied.

Many studies on endometriosis recruit participants with the classic description of disease and use pelvic pain as the surrogate marker of endometriosis recurrence or treatment failure. But pain is not always

Conflict of interest: The author has declared that no conflict of interest exists.

from endometriosis, is subjective, and is commonly multifactorial. Therefore, using "pain" as the study outcome that equals endometriosis is flawed. Some studies do not even require histologic confirmation of endometriosis for inclusion, despite this being the gold standard for diagnosis. Additionally, a narrow study focus on patients with the classic clinical presentation limits the scientific applicability to a much larger population of women with endometriosis with variable phenotypes.

The stages of endometriosis refer to the extent of intra-abdominal disease noted surgically but does not correlate with symptoms, such that patients with extensive pelvic disease may present with mild symptoms, and those with minimal pelvic disease may present with severe pain. For clinicians treating endometriosis, there is often a recognition that, functionally, two types of endometriosis exist. Stage 1 to 2 (mild) disease and stage 3 to 4 (severe) disease have very distinct manifestations, both intraoperatively and clinically. It is almost as if endometriosis is a binary process, rather than a spectrum of progressive stages. The stages should not be grouped and studied as one disease process. Until we recognize the fatal flaws in our research design and change our methods, we will continue to stymie our own progress.

\section{Treatment of endometriosis}

We currently have an adequate armamentarium for the medical treatment of endometriosis, including combined estrogen and progestin contraceptives, progestin-only methods, gonadotrophin-releasing hormone (GnRH) agonists, and GnRH antagonists. Contrary to popular belief, no medical therapy has ever been found to be more effective than another for the treatment of the pain associated with endometriosis (1).

We commonly begin with a hormonal contraceptive for the presumptive treatment of endometriosis when treating a patient with a suggestive history. If hormonal contraceptives are not effective or 
no longer provide relief, when is it time to consider surgery for treatment? Data suggest that offering a GnRH agonist at this juncture improves pain and has short-term cost effectiveness $(7,8)$. While I understand these arguments, as the surgeon who sees countless patients who have suffered for months, sometimes years, following one medical therapy after another, I think it's time to question this approach. We potentially deny or delay the treatment patients need. Some patients with endometriosis will benefit from cytoreductive surgery (9). And for patients with little to no endometriosis, the disservice continues by "treating" them with medications that have substantial adverse side effects for prolonged periods for possibly the wrong diagnosis, and/or by not addressing their true source of pain.

\section{Surgery for endometriosis}

Although I am a strong proponent of excision of endometriosis (physically cutting out) and teach this method to my trainees, there are still limited high-quality data to suggest that excision is superior to ablation (destruction of tissue by use of electrocautery or laser) for the treatment of pain (10). Using pain relief as a surrogate for the success of the excision versus ablation surgery misses many of the other key characteristics of endometriosis that affect the patient.

First, consider the diagnosis of disease. Endometriosis can have a variable and often subtle appearance. In part, because of this variability, the positive predictive value of visual inspection may be approximately 60\% (11). Therefore, biopsy with histopathologic confirmation is necessary for diagnosis. Many surgeons are not familiar with the variable and often subtle appearance of endometriosis, leading to misdiagnosis, i.e., true endometriosis may be missed, and other nonendometriosis lesions may be misdiagnosed as disease. Excision of endometriosis or, at minimum, a biopsy of lesions, must be performed for a proper diagnosis. This is an important fact that we must impart to our trainees. Further, it should never come as a surprise that endometriosis is found near the ureters, bowel, and bladder. Thus, the surgeon should come to the operating room prepared and equipped to treat. The surgical skill of the surgeon should not be the rate-limiting step in a patient's progress.

The laparoscopy-with-biopsy approach captures many patients with endometriosis, but this method does not apply to those who present with deeply infiltrating disease, ovarian endometriomas, or other advanced disease. These diseases can frequently be diagnosed preoperatively by appropriate imaging techniques. Surgeries for advanced-stage endometriosis are among the most difficult, technically challenging procedures to perform and may lead to serious complications, with adverse consequences for long-term health and fertility. The surgeon must be able to recognize the case complexity preoperatively and take measures to maximize the clinical outcome. But how many surgeons can treat these more unique types of advanced disease? It may be time to examine our administration of care in the United States and consider novel treatment approaches. The British Society for Gynaecological Endoscopy (BSGE) has worked to create approved endometriosis centers where women - particularly those with severe disease - are sent to providers who can administer high-quality surgical care. Their work is continuously scrutinized and held to the highest standards. This forward-thinking approach is helping women receive the excellent clinical outcomes they deserve (12). Women in the United States would benefit from broader access to highly skilled and experienced surgeons specializing in endometriosis.

For the benefit of our patients, it is time to change our teaching and our thinking about "classic endometriosis." What is taught is not wrong, but it is just a snippet of the entire endometriosis disease spec- trum. Forward progress in research and treatment will continue to suffer until we embrace the variability in clinical presentation. We must better educate clinicians and improve our diagnosis and management of the disease. We must improve surgical training for treatment and refer patients to specialist care for surgery when appropriate. As a medical community, it is time to rethink endometriosis and adapt our practices for the benefit of our patients.

Address correspondence to: Kristin Patzkowsky, Department of Gynecology and Obstetrics, 600 N. Wolfe St., Phipps 249, Johns Hopkins School of Medicine, Baltimore, Maryland, USA. Phone: 410.614.4495; Email: Kpatzko1@jhmi.edu.

1. Practice bulletin no. 114: management of endometriosis. Obstet Gynecol. 2010;116(1):223-236.

2. Benagiano $\mathrm{G}$, et al. The history of endometriosis. Gynecol Obstet Invest. 2014; 78(1):1-9.

3. Russel WW. Aberrant portins of the Mullerian duct found in an ovary. Johns Hopkins Med J. 1899;10(1):8-10.

4. Sampson JA. Perforating hemorrhagic (chocolate) cysts of the ovary. Arch Surg. 1921;3(2):245-323.

5. Till S, et al. Psychology of chronic pelvic pain: prevalence, neurobiological vulnerabilities, and treatment. Clin Obstet Gynecol. 2019;62(1):22-36.

6. Howard FM. Endometriosis and mechanisms of pelvic pain. J Minim Invasive Gynecol. 2009;16(5):540-550.

7. Ling FW. Randomized controlled trial of depo leuprolide in patients with chronic pelvic pain and clinically suspected endometriosis. Pelvic Pain Study Group. Obstet Gynecol. 1999;93(1):51-58.

8. Winkel CA. A cost-effective approach to the management of endometriosis. Curr Opin Obstet Gynecol. 2000;12(4):317-320.

9. Abbot J, et al. Laparoscopic excision of endometriosis: a randomized, placebo-controlled trial. Fertil Steril. 2004;82(4):878-884.

10. Pundir J, et al. Laparoscopic excision versus ablation for endometriosis-associated pain: an updated systematic review and meta-analysis. J Minim Invasive Gynecol. 2017;24(5):747-756.

11. Walter AJ, et al. Endometriosis: correlation between histologic and visual findings at laparoscopy. Am JObstet Gynecol. 2001;187(7):1407-1411.

12. Byrne D, et al. Laparoscopic excision of deep rectovaginal endometriosis in BSGE endometriosis centres: a multicentre prospective cohort study. BMJ Open. 2018;8(4):e018924. 